\title{
A Stereological Description of Size and Number of Zona Fasciculata Mitochondria in Rat Adrenal Cortex after Hypophysectomy*
}

\author{
Shigetaka SEKIYAMA, Nagasumi YAGO, Yuko IWAI, \\ Hiromi KUROKAWA, FUMIAKI SATO** AND AKIHIRo SHIRAGAI*** \\ Divisions of Physiology and Pathology, Radiation Hazards** and Physics***, \\ National Institute of Radiological Sciences, Chiba 280.
}

\begin{abstract}
Synopsis
The volume fractions and wet weights of the three cortical layers and medulla of the rat adrenal gland have been quantitatively determined by the stereological volumetry on the histological photograph during the period of 3 days following hypophysectomy. Both of the volume fraction and wet weight decreased significantly only in the zona fasciculata after hypophysectomy. The mitochondria in the parenchymal cells of the zona fasciculata have been reconstructed three-dimensionally by Bach's theory on sphere size distribution. The total number of the mitochondria in the unit cytoplasmic volume and the average values of their radius and volume have been computed. The size distribution histograms have also been obtained in terms of the relative frequency as a function of the mitochondrial radius. These quantitative stereological computations have offered the evidences that 1 ) there is a volume increase in the zona fasciculata mitochondria following hypophysectomy, and 2) the total number of the mitochondria in an individual rat decreased to the half in about 3 days.
\end{abstract}

The biochemical components of the adrenocortical mitochondria in the hypophysectomized rat have been quantitatively analysed by Harding and Nelson (1964a, b) and Kimura (1969). On the other hand, the fine structural changes in these mitochondria have not been quantitatively determined, although many investigators have studied electron microscopically. (see Fawcett et al., 1969, for review). The ultrastructural changes of these mitochondria must be, therefore, prosecuted in quantitative ways to study the correlation between the biochemical and morphological characteristics.

In our previous study (Yago et al., 1971), we have analysed the changes in the geometrical characteristics of the zona fasciculata mitochondria in the rat adrenal cortex after ACTH administration by the use of Bach's

Received for publication July 13, 1971.

*This work was supported in part by Grant A-7006 from the Ministry of Education. theory on sphere size distribution (1967). The present paper deals with the stereological changes in size and number of the zona fasciculata mitochondria during the period of 3 days following hypophysectomy.

\section{Materials and Methods}

Male Wistar rats weighing about $250 \mathrm{~g}$ were hypophysectomized via a transaural route, and sacrificed at 24,48 , and $72 \mathrm{hr}$ after the operation. Intact rats served as control. All animals were maintained on a balanced diet in an air-conditioned room at $27^{\circ} \mathrm{C}$. For the determination of the volume fractions of the histological constituents of adrenal gland after hypophysectomy, five rats were sacrificed in each of the rat-groups. All adrenals excised from these rats in the same group were bisected, and fixed together in $4 \%$ formalin in $0.1 \mathrm{M}$ sodium phosphate buffer $(\mathrm{pH}$ 7.4). Then, four bisected tissues were taken at random from individual animal groups, made into semi-serial paraffin sections, $4 \mu$ thick with $100 \mu$ intervals, and stained with haematoxylin and eosin. Whole-view pictures of the histological sections, totaling about 
20 to 30 in number, were taken from each bisected tissue at a primary magnification of $400 \times$. The volume fractions of the three cortical zones and medulla were deduced from point-counting volumetry (Weibel et al., 1966) on the enlarged photographs, respectively.

Procedures for recording electron micrographs and for computing the values of stereological parameters of the mitochondria in parenchymal cells of the zona fasciculata were the same as described previously (Yago et al., 1971). In brief, the mitochondrial populations in individual animal groups were theoretically reconstructed by incorporating into Bach's theory the values of diameters of about 500 profiles of the mitochondria obtained from 20 or more enlarged micrographs sampled at random in each experimental group. Other details were the same as described in the previous paper (Yago et al., 1971).

\section{Results}

The volume fraction of the zona fasciculata decreased slightly but significantly after hypophysectomy from the average value of 0.65 in the intact control to that of 0.55 in the hypophysectomized rats at $72 \mathrm{hr}$ (Fig. 1). The

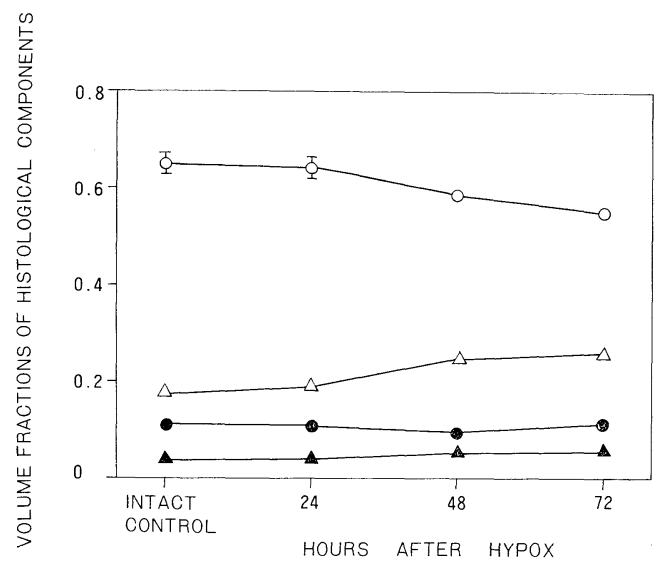

Fig. 1. Changes in the volume fractions of the histological constituents (three cortical layers and medulla) in the rat adrenal gland following hypophysectomy (abbreviated as hypox). Values are means \pm standard error of the mean calculated from the four values in each of the animal groups. In some of the determinations the values of the standard error were so small as to be hidden in the marks. Zona fasciculata, - $\bigcirc-$; zona glomerulosa, $-\triangle-$; zona reticularis, - - ; and medulla, $-\mathbf{A}-$. weights of individual histological components have been calculated by multiplying the wet weight of the whole adrenal gland with the values of the volume fractions. It is seen in Figure 2 that the weight of the zona fasciculata alone showed a significant desrease after hypophysectomy.

Values of the stereological parameters for the mitochondrial population in the parenchymal cells of the zona fasciculata are presented in Table 1. The mitochondrial population at $24 \mathrm{hr}$ was not analysed in the present study, because there were no significant changes in both the volume fraction and weight of the zona fasciculata at this period. It is conceivable that the average size of these mitochondria at 48 and $72 \mathrm{hr}$ was larger than in the control, respectively. Thus, the average volume $(\bar{V})$ at $72 \mathrm{hr}$ was nearly $40 \%$ over the control. Computation of the size distribution in terms of the relative frequency as a function of radii of the reconstructed mitochondria (Fig. 3) has revealed that there is a shift of the mitochondrial population to the right on the abscissa after hypophysectomy. The values of mitochondrial volume fractions

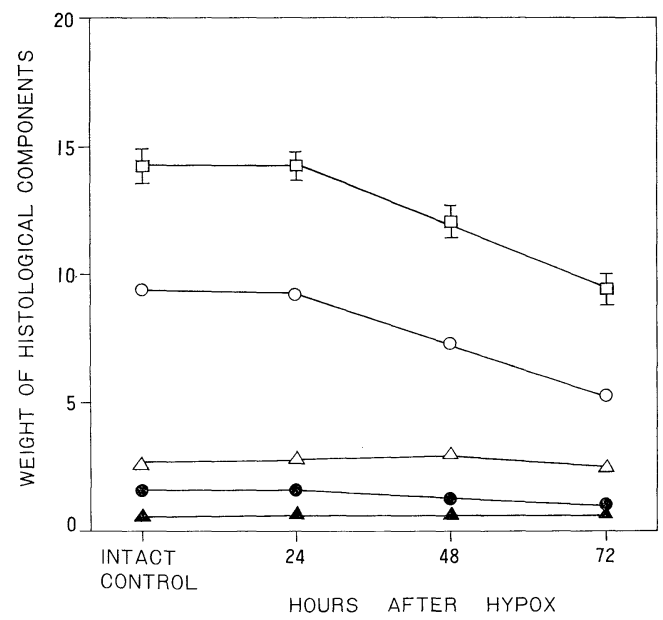

Fig. 2. Weight changes in the histological constituents (three cortical layers and medulla) in the rat adrenal gland following hypophysectomy. Values are expressed in terms of $\mathrm{mg} / 100 \mathrm{~g}$ body weight. Whole gland, - $\square-$ Other details are the same as in Fig. 1. 
Table 1. Changes in the stereological parameters of the zona fasciculata mitochondria in the rat adrenal cortex following hypophysectomy

\begin{tabular}{ccccc}
\hline $\begin{array}{c}\text { Hours after } \\
\text { hypophysectomy }\end{array}$ & $\begin{array}{c}\text { Average radius } \\
\bar{R}(\mu)\end{array}$ & $\begin{array}{c}\text { Average volume } \\
\bar{V}\left(\mu^{3}\right)\end{array}$ & $\begin{array}{c}\text { Average } \\
\text { number } N_{V}\end{array}$ & $\begin{array}{c}\text { Volume } \\
\text { fraction } V_{V}\end{array}$ \\
\hline Intact control & 0.469 & 0.617 & 694 & 0.428 \\
48 & 0.508 & 0.690 & 729 & $\begin{array}{c}(0.370) \\
0.484 \\
(0.503) \\
0.448 \\
(0.487)\end{array}$ \\
\hline
\end{tabular}

These values have been computed by Bach' theory directly based on the distribution of profile radii (see Fig. 3). Values for intact controls are taken from the previous paper (Yago et al., 1971). The $V_{V^{-}}$ values shown in the parentheses indicate the results of point-counting volumetry for the volume fraction of cytoplasm occupied by the mitochondrial population. The $N_{V}$-values have been expressed in terms of the number of mitochondria per $1,000 \mu^{3}$ of the cytoplasm of the parenchymal cells in the zona fasciculata.

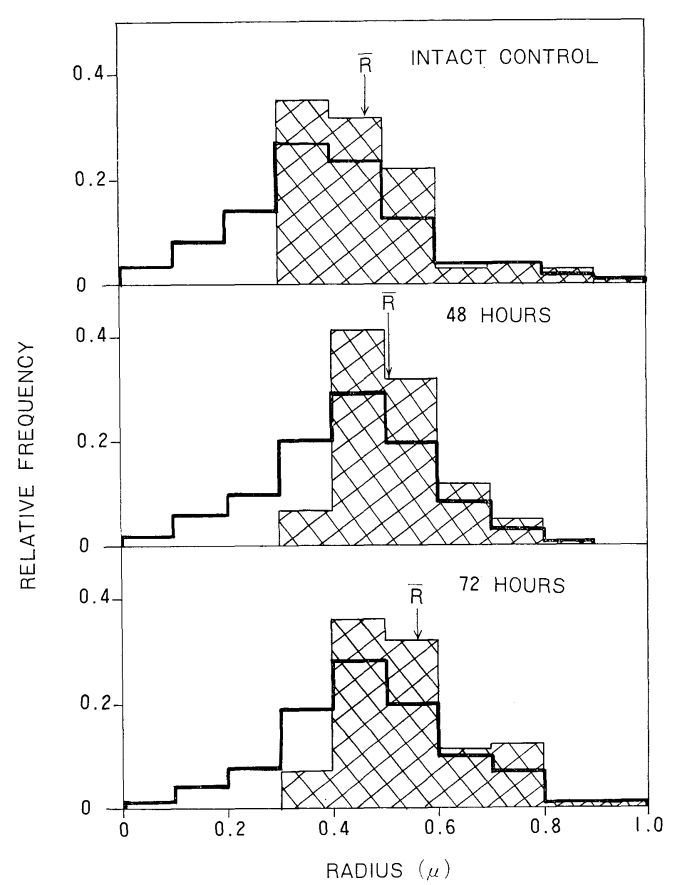

Fig. 3. Size distribution histograms of the reconstructed mitochondrial population (hatched histograms). The computation was performed by Bach's theory based on the continuous distribution of spheres. The histograms drawn with solid lines refer to the size distribution of mitochondrial profiles. The histograms for intact controls have been taken from our previous paper (Yago et al., 1971). The $\chi^{2}$-test for the difference in the size distribution histograms between control and hypophysectomized groups showed it to be highly significant $(\mathrm{p}<0.01)$.
$\left(V_{V}\right)$ obtained by the application of Bach's theory and by point-counting volumetry coincided very well with each other throughout the experimental periods (Table 1).

To calculate the total cytoplasmic volume of parenchymal cells in the zona fasciculata in an individual rat, weights of the zona fasciculata (Fig. 2) were converted into the volumes with the specific weight assumed to be unity. Then the volumes were multiplied with a factor of 0.875 to finally obtain the volumes of the total cytoplasmic volume. The factor of 0.875 has been obtained in our laboratory by the planimetric volumetry for the cytoplasmic volume fraction of parenchymal cells in the zona fasciculata in intact controls and taken in the present study as a constant. By multiplying the values of the cytoplasmic volume with the $N_{V}$-values (Table $1)$, the total number of the mitochondria in the zona fasciculata in an individual rat was obtained. As illustrated in Fig. 4, it decreased rapidly after hypophysectomy. Sum of the volumes of mitochondria in the entire zona fasciculata is calculated to be $5.89 \times 10^{9} \mu^{3}$ or $5.89 \mathrm{~mm}^{3}$ per rat in the control and 3.68 $\mathrm{mm}^{3}$ in the hypophysectomized rats at $72 \mathrm{hr}$.

\section{Discussion}

A significant decrease in the weight of the 


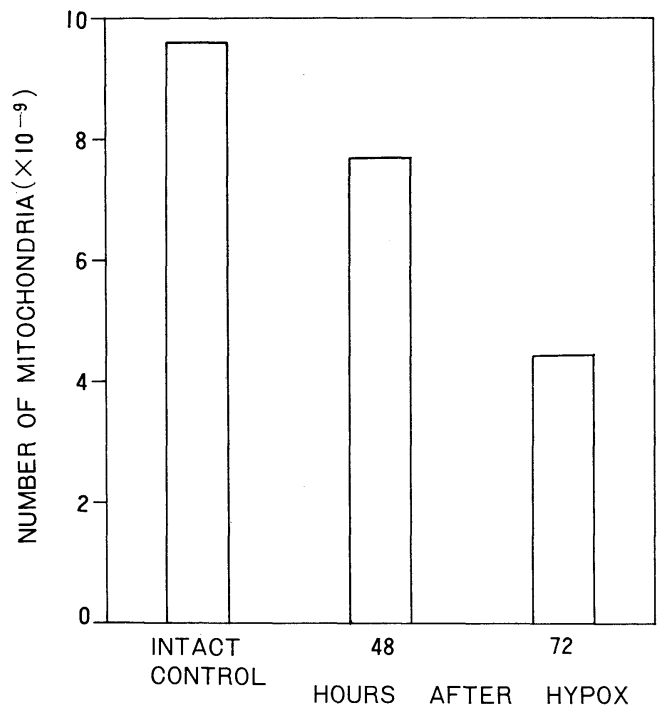

Fig. 4. The total number of the zona fasciculata mitochondria in an individual rat. Values are expressed in terms of the number of mitochondria per $100 \mathrm{~g}$ body weight.

zona fasciculata of rat adrenal following hypophysectomy forms quite a contrast to the $50 \%$ increase $72 \mathrm{hr}$ after the whole-body exposure to the $\mathrm{X}$-irradiation of a lethal dose of $1,000 \mathrm{R}$ (unpublished). These responses of the zona fasciculata would be taken as the quantitative morphological evidence supporting the hypothesis that the zona fasciculata is the major site for the corticosterone biosynthesis in intact rats (Baniukiewicz et al., 1968).

We have already reported (Yago et al., 1971) that ACTH induces an increase in the mitochondrial volume in the zona fasciculata when acutely administered into the rat. However, the mitochondrial volume increase detected in the present study must be quite different from that which can be observed after ACTH administration. It may be predicted that a small but definite amount of $\mathrm{ACTH}$ is required for maintaining the structural equilibrium (Loud, 1968) of these mitochondria and hypophysectomy results in a perturbation in this equilibrium. The fact that the sensitivity of the rat adrenal cortex in response to ACTH decreases progressively after hypophysectomy (Singer and StackDunne, 1955; Vernikos-Danelli, 1969) may be relevant to the perturbation referred to above.

Since the total volume of the mitochondria in the zona fasciculata in an individual rat was not preserved but significantly decreased after hypophysectomy, the decrease in the total number of mitochondria may not be explained on the basis of a simple fusion, if any, between mitochondria. Moreover, the process of decrease may be considered to be grossly parallel to those in the enzymatic activities of adrenocortical mitochondria, viz. succinic dehydrogenase and cholesterol sidechain desmolase, during the corresponding period after hypophysectomy (Kimura, 1969). Therefore, it would be possible that the process for the genesis of the zona fasciculata mitochondria is dependent on the presence of $\mathrm{ACTH}$, as originally suggested by Kahri (1968). Nussdorfer and Mazzocchi (1970) have shown that the number of mitochondria of the zona fasciculata per cell decreased in the rats that were injected with corticosterone in an attempt to inhibit the ACTH secretion from the pituitory. These considerations strengthen the speculation that the decrease in the total number of the mitochondria in the zona fasciculata after hypophysectomy may reflect the decay process of the mitochondrial population. Since Figure 4 does not indicate a first-order process, the kinetics remain to be elucidated. However, it may be noted here that the total number decreased to the half in about 3 days following hypophysectomy. This would suggest that the mitochondria in the zona fasciculata are extremely labile in the absence of pituitary, since the half-life of rat liver mitochondria has been measured to be about 10 days (Fletcher and Sanadi, 1961; Bailey et al., 1967).

With respect to the validity of applying Bach's theory to our purposes, we have found that the values of mitochondrial volume fractions $\left(V_{V}\right)$ determined by the two independent methods coincided very well with 
each other (Table 1). Therefore, it is reasonable to conclude that Bach's theory may be applied to the analyses of the mitochondrial population in the zona fasciculata at least within the period of 3 days after hypophysectomy. In addition, it should be very difficult to detect, by visual inspection of electron micrographs, any difference in size and number of mitochondria between the control and experimental rat groups (Fig. 3), because even the two-dimensional size distributation patterns of profile-radii were very similar to each other. Further, in such cases it must be impossible to deduce any conclusion about the three-dimensional size distribution by an intuition. One of the examples of effective use of quantitative stereological methods has thus been well illustrated in the computations of size distributions.

\section{Acknowledgement}

We wish to thank Mr. K. Fukuhisa and Misses E. Oshi and A. Onuma, Data Processing Laboratory of our institute, for their generous help in the use of the electronic computer.

\section{References}

Bach, G. Quantitative methods in morphology. (edited by E. R. Weibel and H. Elias). Springer, New York, p. 23 (1967).
Bailey, E., C. B. Taylor and W. Bartley (1967). Biochem. J. 104, 1026.

Baniukiewicz, S., A. Brodie, C. Flood, M. Motta, M. Okamoto, J. F. Tait, A. S. Denton, J. R. Goding, B. A. Scoggins, E. M. Wintour and R. D. Wright. Functions of the adrenal cortex. (edited by K. W. McKerns). North Holland Publishing Co., Amsterdam. Vol. 1, p. 153 (1968).

Fawcett, D. W., J. A. Long and A. L. Jones. Recent Progress in Hormone Research. (edited by E. B. Astwood). Academic Press, New York. Vol. 25, p. 315 (1969).

Fletcher, M. J. and D. R. Sanadi (1961). Biochim. Biophys. Acta 51, 356.

Harding, B. W. and D. H. Nelson (1964a). Endocrinology 75, 501.

Harding, B. W. and D. H. Nelson (1964b). Ibid. 75, 506.

Kahri, A. I. (1958). J. Cell Biol. 36, 181. Kimura, T. (1969). Endocrinology 85, 492. Loud, A. V. (1968). J. Cell Biol. 37, 27.

Nussdorfer, G. G. and G. Mazzocchi (1970). Z. Zellforsch. 111, 90.

Singer, B. and M. P. Stack-Dunne (1955). J. Endocrinol. 12, 130.

Vernikos-Danellis, J. (1969). Endocrinology 84, 1507.

Weibel, E. R., G. S. Kistler and W. F. Scerle (1966). J. Cell Biol. 30, 23.

Yago, N., S. Sekiyama, H. Kurokawa, Y. Iwai, F. Sato, and A. Shiragai (1971). Endocrinol. Japon. 18, 359. 